\title{
Correction to: The impact of complications after initial prostate biopsy on repeat protocol biopsy acceptance rate. Results from the Prostate Cancer Research International: Active Surveillance JAPAN study
}

\author{
Yoichiro Tohi ${ }^{1}$ (1) Takuma Kato ${ }^{1} \cdot$ Ryuji Matsumoto $^{2} \cdot$ Nobuo Shinohara $^{2} \cdot$ Kenichiro Shiga $^{3} \cdot$ Akira Yokomizo $^{3}$. \\ Masaki Nakamura $^{4} \cdot$ Haruki Kume $^{4} \cdot$ Koji Mitsuzuka $^{5} \cdot$ Hiroshi Sasaki $^{6} \cdot$ Shin Egawa $^{6} \cdot$ Masafumi Matsumura $^{7}$. \\ Katsuyoshi Hashine ${ }^{7} \cdot$ Junichi Inokuchi ${ }^{8} \cdot$ Masatoshi Eto $^{8} \cdot$ Haruki Baba $^{9} \cdot$ Tomohiko Ichikawa $^{9}$. \\ Hidefumi Kinoshita ${ }^{10} \cdot$ Tadashi Matsuda $^{10} \cdot$ Yoshiyuki Kakehi $^{1} \cdot$ Mikio Sugimoto $^{1}$
}

Published online: 3 March 2022

(c) The Author(s) under exclusive licence to Japan Society of Clinical Oncology 2022

\section{Correction to: International Journal of Clinical Oncology https://doi.org/10.1007/s10147-020-01761-3}

In the original publication, Table 4 was published with missing entries. The corrected Table 4 is given in this correction.

The original publication has been corrected.
The original article can be found online at https://doi.org/10.1007/ s10147-020-01761-3.

Yoichiro Tohi

yoto716yotoyoto@gmail.com

1 Department of Urology, Faculty of Medicine, Kagawa University, 1750-1, Ikenobe, Miki-cho, Kita-gun, Kagawa 761-0793, Japan

2 Department of Renal and Genito-Urinary Surgery, Graduate School of Medicine, Hokkaido University, Hokkaido, Japan

3 Division of Urology, Harasanshin Hospital, Fukuoka, Japan

4 Department of Urology, Graduate School of Medicine, The University of Tokyo, Tokyo, Japan

5 Department of Urology, Tohoku University Graduate School of Medicine, Miyagi, Japan
Table 4 Multivariate logistic regression analysis for repeat protocol biopsy acceptance at 1 year by patients' baseline characteristics and complications after the initial biopsy

\begin{tabular}{lll}
\hline Variable & \multicolumn{2}{l}{ Multivariate } \\
\cline { 2 - 3 } & OR $(95 \% \mathrm{CI})$ & $P$-value \\
\hline Age at biopsy & $1.003(0.961-1.048)$ & 0.879 \\
PSA & $0.972(0.799-1.183)$ & 0.78 \\
Prostate volume & $1.004(0.986-1.023)$ & 0.638 \\
Biopsy core & $1.029(0.948-1.117)$ & 0.493 \\
Transperineal biopsy (vs & $1.211(0.668-2.196)$ & 0.528 \\
$\quad$ transrectal approach) & & \\
Complication & $3.415(0.538-21.664)$ & 0.793 \\
Infection & $2.017(0.93-4.374)$ & 0.076 \\
Hematuria & $0.061(0.006-0.574)$ & 0.015 \\
Hematospermia & $4.68(1.864-11.75)$ & 0.001 \\
Pain &
\end{tabular}

$O R$ odds ratio, $C I$ confidence interval

Publisher's Note Springer Nature remains neutral with regard to jurisdictional claims in published maps and institutional affiliations.

6 Department of Urology, Jikei University School of Medicine, Tokyo, Japan

7 Department of Urology, Shikoku Cancer Center, Ehime, Japan

8 Department of Urology, Graduate School of Medical Sciences, Kyushu University, Fukuoka, Japan

9 Department of Urology, Graduate School of Medicine, Chiba University, Chiba, Japan

10 Department of Urology and Andrology, General Medical Center, Kansai Medical University, Osaka, Japan 